\title{
Infrequently Performed Studies in Nuclear Medicine: Part 2*
}

\author{
Anita MacDonald and Steven Burrell \\ Department of Diagnostic Radiology, Queen Elizabeth II Health Sciences Centre and Dalhousie University, Halifax, Nova Scotia, \\ Canada
}

Nuclear medicine is a diverse medical field, encompassing a wide range of studies. Many studies are performed on a daily basis, whereas some nuclear medicine studies are performed on a more infrequent basis. The following article is the second article in a 2-part series on infrequently performed studies in nuclear medicine. The procedures reviewed include CSF shunt imaging, brain death scintigraphy, testicular scanning, quantitative lung perfusion studies, lymphoscintigraphy, and salivary gland scintigraphy. This article may be of use to technologists faced with the task of performing one of these uncommon studies. After reviewing the article, the reader should be able to list the indications for each study, list the recommended doses, describe the proper method of dose administration for each study, discuss patient preparation for each procedure, and describe issues that may arise when performing these studies.

Key Words: infrequently performed studies; CSF shunt; brain death; testicular scan; quantitative lung perfusion; lymphoscintigraphy; salivary gland scintigraphy

J Nucl Med Technol 2009; 37:1-13

DOI: 10.2967/jnmt.108.057851

$\mathbf{T}$ he following article is Part 2 in a 2-part series on infrequently performed studies in nuclear medicine. As discussed in Part 1 (1), many nuclear medicine studies are performed daily and cover a wide range of areas in the field of medicine. However, there are also several studies that are performed less frequently, and it may be difficult to maintain skills in studies that are infrequently performed. This article provides a review of 6 such studies: cerebral spinal fluid (CSF) shunt imaging, brain death scintigraphy, testicular scanning, quantitative lung perfusion studies, lymphoscintigraphy, and salivary gland scintigraphy. The

\footnotetext{
Received Sep. 4, 2008; revision accepted Nov. 17, 2008.

For correspondence or reprints contact: Anita MacDonald, Department of Diagnostic Imaging, Room 3016, 3 South Victoria, VG Site, Queen Elizabeth II Health Sciences Centre and Dalhousie University, 1278 Tower Rd., Halifax, Nova Scotia, Canada B3H 2 Y9.

E-mail: ajmacdon@dal.ca

*NOTE: FOR CE CREDIT, YOU CAN ACCESS THIS ACTIVITY THROUGH THE SNM WEB SITE (http://www.snm.org/ce_online) THROUGH MARCH 2011.

COPYRIGHT (C) 2009 by the Society of Nuclear Medicine, Inc.
}

clinical and technical aspects of each study, along with a brief comparison of alternative modalities that may also be used, are discussed.

\section{CSF SHUNT IMAGING}

Hydrocephalus is an overaccumulation of CSF in the ventricles of the brain. Many reasons exist for the development of hydrocephalus in a patient, including an overproduction of CSF, an obstruction of CSF flow, and problems with reabsorption of the CSF. Broadly speaking, hydrocephalus may be classified as communicating hydrocephalus and noncommunicating hydrocephalus.

Communicating hydrocephalus occurs when there is patent communication between the ventricles but impairment of CSF reabsorption. Noncommunicating hydrocephalus is caused by an obstruction of the CSF flow out of the ventricles. Both types may lead to an increase in intracranial pressure and mental instability and many other symptoms such as urinary incontinence, gait instability, headaches, and coma.

CSF shunts are inserted to treat the symptoms of hydrocephalus. Two types of CSF shunts are the ventriculoperitoneal (ventricle to peritoneal cavity) shunt and the ventriculoatrial (ventricle to right atrium) shunt. The shunt system consists of a reservoir with a valve placed beneath the soft tissues of the head, a proximal (afferent) tubing line leading from one of the ventricles to the reservoir, and a distal (efferent) tubing line leading from the reservoir to the peritoneal cavity (Fig. 1) or atrium. The outer side of the reservoir is covered by a pliable material that can be depressed manually to pump the reservoir and punctured with a needle. Hydrocephalus-related symptoms that worsen after shunt placement may indicate a malfunctioning or obstructed shunt. A malfunctioning shunt may be caused by a mechanical issue such as disconnection or kinking of the shunt tubing. Shunt obstructions may be caused by occlusion of the tip of the tubing by the omentum in the peritoneal cavity or emboli in the shunt tubing. Nuclear medicine CSF shunt imaging is used to determine whether the shunt is patent and to exclude shunt obstruction.

${ }^{99 \mathrm{~m}}$ Tc-diethylenetriaminepentaacetic acid ( $\left.{ }^{99 \mathrm{~m}} \mathrm{Tc}-\mathrm{DTPA}\right)$, ${ }^{111} \mathrm{In}-\mathrm{DTPA}$, and ${ }^{99 \mathrm{~m}} \mathrm{Tc}$-pertechnetate are radiopharmaceuticals that may be used for CSF shunt studies. The ${ }^{99 \mathrm{~m} T \mathrm{Tc}}$ 


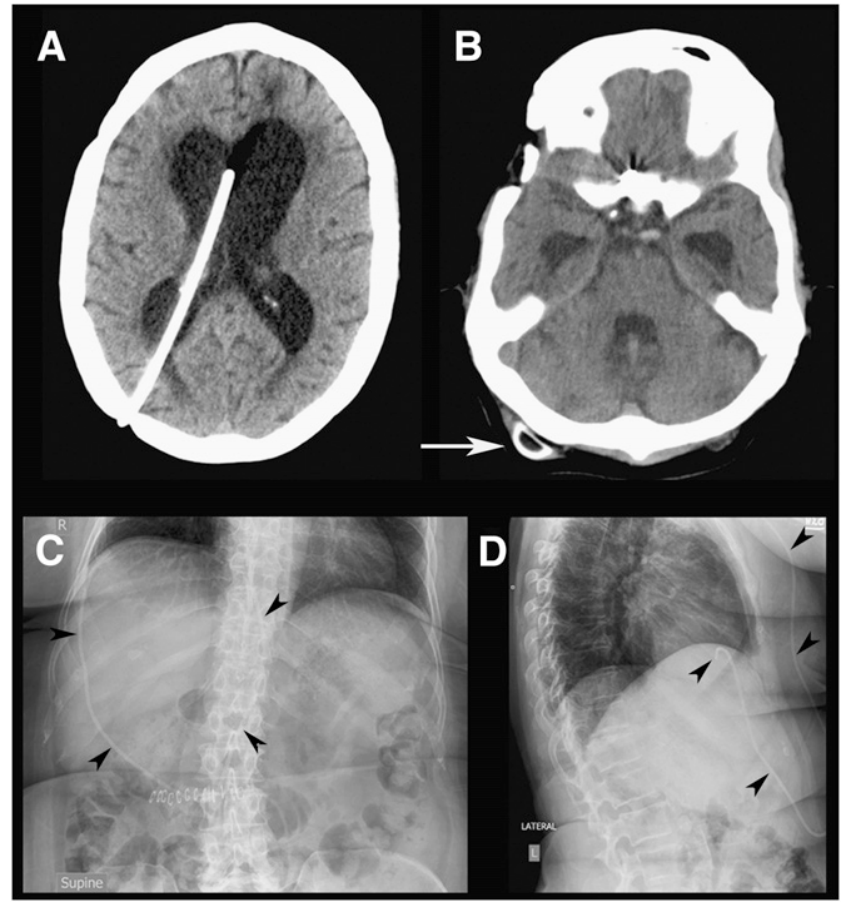

FIGURE 1. Anatomic imaging in questioned obstructed shunt. (A) Axial slice from CT scan demonstrates enlarged lateral ventricles and proximal limb of CSF shunt. (B) CT slice more inferiorly shows shunt port (arrow). Frontal (C) and lateral (D) radiographs of lower chest and upper abdomen demonstrate shunt tubing (arrowheads), terminating in right upper quadrant of abdomen.

tracers are generally preferred as the study is usually completed within a few hours, so the longer half-life of ${ }^{111}$ In is not required.

The recommended dose of $99 \mathrm{~m}$ Tc-DTPA is approximately $18.5 \mathrm{MBq}(\sim 0.5 \mathrm{mCi})$ in $0.3-0.5 \mathrm{~mL}$ (2). The small volume is used to ensure minimal disruption of the normal CSF flow physiology.

The dose is aseptically injected by the physician into the CSF shunt reservoir, with the patient in the supine position. The shunt reservoir is felt as a raised area on the scalp, just below the skin and superficial to the bone. The specifics of the injection will depend on the physician performing the procedure. Many will first measure the CSF opening pressure using a manometer on accessing the reservoir with the needle. Once the opening pressure has been measured, the radiopharmaceutical is injected.

Immediately after injection, the patient is imaged in the supine position, with dynamic imaging at a rate of $1 \mathrm{~min} /$ frame to include the injection site at the shunt reservoir to the location of the end tip of the distal tubing of the shunt. For ventriculoatrial shunts, this would include imaging from the head to the heart, and for ventriculoperitoneal shunts imaging would include the head to the peritoneal cavity. With a camera equipped with a small field of view, several dynamic studies or static views after injection of the shunt reservoir may be required, and, correspondingly, the patient or camera may need to be moved until the end location of the radioactivity in the tubing has been reached. Lateral images may also be obtained of the head to visualize reflux into the ventricles. Imaging may be considered with the patient in the upright position to increase hydrostatic pressure and therefore encourage CSF flow. This procedure may be especially helpful to perform when supine imaging has not been successful and to help further negate a false-positive study.

During injection, some physicians may manually occlude the distal limb of the tubing where it leaves the reservoir to help reflux a portion of the radiopharmaceutical into the ventricles, thereby assessing patency of the proximal limb of the tubing. Other physicians choose not to do so, preferring to see if there is reflux under more physiologic conditions. If there is no reflux of radiopharmaceutical into the ventricles after occlusion during shunt injection, there may be an obstruction of the proximal portion of the CSF shunt tubing. However, to not visualize the ventricles does not necessarily imply an occlusion. For example, some shunts are equipped with a flap valve that will not allow passage of the radioactivity into the ventricles if the tracer is administered distal to the flap valve. Assessment of the patency of the proximal tubing is reliably achieved with the measurement of the opening pressure: a normal or elevated pressure in the reservoir implies patency of the proximal tubing.

Most obstructions occur in the distal limb. In ventriculoatrial shunts, activity must be visualized in the heart for the study to be normal and the distal limb to be deemed patent. If ${ }^{99 \mathrm{~m}} \mathrm{Tc}-\mathrm{DTPA}$ is used, visualization of the kidneys implies at least some degree of shunt patency. In ventriculoperitoneal shunts, activity must be diffusely visualized in the abdomen for the study to be considered normal (Fig. 2). Figure 3 displays an example of an obstructed ventriculo-

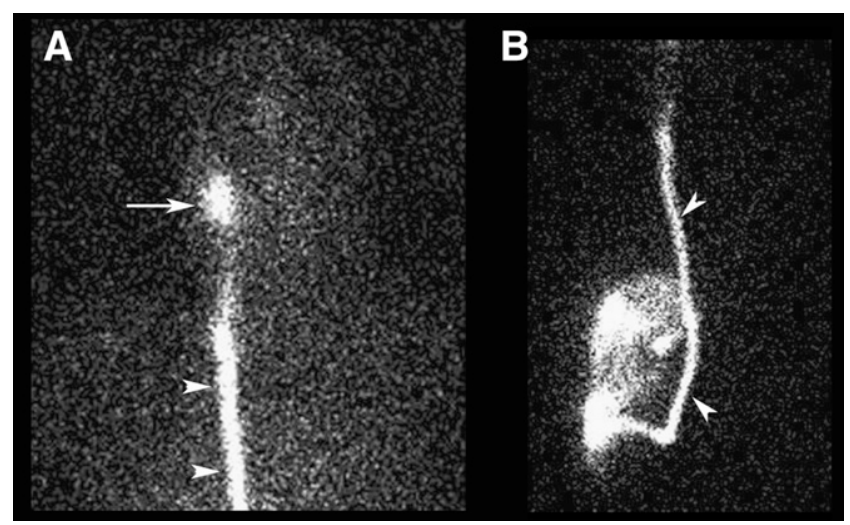

FIGURE 2. Normal CSF shunt study in same patient as in Figure 1. (A) Anterior image of head and chest demonstrates activity concentrated in shunt reservoir (arrow) and within efferent tubing at level of chest (arrowheads). (B) Anterior image of chest and abdomen (smaller scale than A) reveals activity along entire course of tubing (arrowheads), ultimately spilling freely into abdominal cavity. 


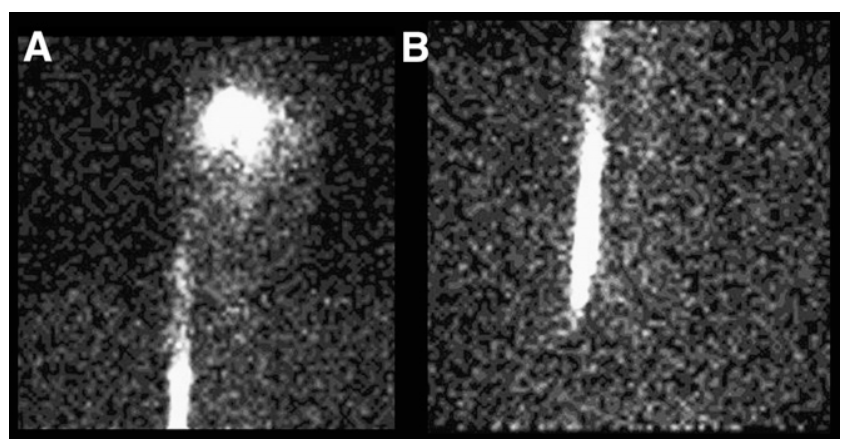

FIGURE 3. Obstructed CSF shunt. (A) Anterior image at level of head and chest demonstrates reflux into ventricles of brain and transit down efferent limb of shunt. (B) Anterior image at level of lower chest and upper abdomen reveals abrupt termination of activity in shunt tubing at level of lower chest. No spill of activity into abdomen, even on delayed views, was observed.

peritoneal shunt for which serial images were taken with no radioactivity visualized in the peritoneal cavity. Imaging was performed again at the 3- and 24-h marks, demonstrating an obstructed shunt at the lower thoracic level.

In addition to an assessment of "obstructed" versus "not obstructed," some have advocated a more refined analysis assessing the flow velocity of CSF from the reservoir to determine whether there is altered physiology as might arise from a partial obstruction. In this technique (3), a dynamic study is performed with 30 -s images for $15 \mathrm{~min}$. A region of interest (ROI) is drawn around the activity in the reservoir and a time-activity curve is generated. The flow velocity is then calculated on the basis of the rate of disappearance of activity from the reservoir, modeled using an exponential relationship, and the volume of the reservoir. This technique is not universally used.

The CSF shunt study provides a simple, effective, and low-radiation-dose method of assessing CSF shunt patency. However, this method is not frequently requested. Initial assessment of the patient with a CSF shunt presenting with symptoms is performed clinically. The clinician can perform a simple assessment of the shunt system by depressing the bulb of the reservoir. If the bulb readily depresses, the distal limb is likely patent, and if it readily refills, the proximal limb is likely patent. The clinician may also access the shunt reservoir to measure opening pressure and obtain CSF for culture and laboratory analysis. CT is often performed initially to assess ventricular size. This technique is not entirely reliable, however, as patients may have chronically enlarged ventricles. CT is most reliable in this setting if there are prior scans to allow determination of whether the ventricles have increased in size. The CT may also be used to verify the correct placement of a CSF shunt. Routine radiography may be performed to examine the tubing to check for kinking or disconnection. However, when doubt persists, the nuclear medicine CSF shunt study remains a reliable means of assessing shunt patency.

\section{BRAIN DEATH SCINTIGRAPHY}

Brain death is defined slightly differently by various organizations, but the overlying concept is of complete and irreversible loss of function of the brain (4). The term is usually applied in the context of continued function of other organ systems, such as the cardiovascular system. In this scenario, an accurate and timely determination of brain death is necessary to best address the grief and anxiety of family members and to allow timely assessment for possible organ donation. Common causes of brain death are severe traumatic brain injury and subarachnoid hemorrhage (5).

Usually brain death is a clinical diagnosis. Although specific criteria vary among organizations and jurisdictions, an example (paraphrased) includes an established etiology capable of causing neurologic death in the absence of reversible conditions capable of mimicking neurologic death, deep unresponsive coma with absent motor reflexes, absent brain stem reflexes, absent respiratory effort based on the apnea test, and absent confounding factors (such as unresuscitated shock, hypothermia, and certain drug intoxications) (5). In some instances, it may be extremely difficult or even impossible to perform all of the tests required-for example, in severe facial injuries or pulmonary disease. In addition, these tests may have to be performed on several occasions over a 6- to 24 -h period to have confirmatory results. Ancillary diagnostic testing may be recommended in situations in which a definitive diagnosis cannot be made on clinical grounds or in which an institution deems it necessary as part of its routine protocol.

A nuclear medicine brain death study is a simple and minimally invasive test that may assist in the diagnosis of brain death by assessing brain blood flow. The ventilated patient is transported to the department with a variety of medical staff. In some departments in which mobile scanners are available, the nuclear medicine technologist would bring all the necessary equipment for imaging to the patient's unit. There are 2 general nuclear medicine approaches to assessing brain death. The first uses the nonbrain-specific flow agent ${ }^{99 \mathrm{~m}} \mathrm{Tc}$-DTPA, which does not cross the blood-brain barrier, and the second approach uses the lipophilic brain agent ${ }^{99 \mathrm{~m}} \mathrm{Tc}$-hexamethylpropyleneamine oxime $\left({ }^{99 \mathrm{~m}} \mathrm{Tc}-\mathrm{HMPAO}\right)$ or ${ }^{99 \mathrm{~m}} \mathrm{Tc}$-ethylcysteinate dimer ( $\left.{ }^{99 \mathrm{~m}} \mathrm{Tc}-\mathrm{ECD}\right)$.

When using $99 \mathrm{~m} \mathrm{Tc}-\mathrm{DTPA}$, the recommended dose is $555-740 \mathrm{MBq}(15-20 \mathrm{mCi})(6)$. The patient is positioned under a low-energy, high-resolution collimator with a field of view to include carotids to skull vertex, with the camera anterior and parallel to the patient's face. Positioning the patient and camera in this manner ensures that the study may be assessed properly during a comparison of flow to the left and right side. There is no patient preparation for this study; however, proper ventilation should be in place to prevent changes in cerebral blood flow from hyperventilation, and one must also be aware that high levels of barbiturates may decrease cerebral blood flow (6). Some 
institutions may place a tourniquet around the patient's head (above the eyebrows and ears and around the posterior prominence of head) before beginning the procedure, to diminish scalp blood flow, which may confound assessment of brain flow. A tourniquet should not be used if there is head trauma or there is possibility of causing further injury. It is recommended that an intravenous catheter be used for dose administration, followed by a saline flush, to ensure no dose infiltration and adequate bolus administration. A dynamic study should be performed after intravenous injection, with $1-3 \mathrm{~s} /$ frame for at least $60 \mathrm{~s}(6)$. At our institution, the acquisition is started before the injection is given, to ensure imaging begins before the bolus reaches the carotid arteries. To ensure adequate time during the actual scanning of blood flow, we prefer to set up the dynamic scan for at least 3-5 min. Static imaging (for a minimum of $5 \mathrm{~min} / \mathrm{view}$ in the anterior, left, and right lateral positions) should also be performed, after the dynamic acquisition is complete. A posterior static image also may be acquired but may not be necessary and is usually difficult to obtain.

A normal brain-flow study using ${ }^{99 \mathrm{~m}} \mathrm{Tc}-\mathrm{DTPA}$ (Fig. 4) includes visualization of flow up the carotid arteries and onward to the anterior and middle cerebral arteries (ACA and MCA, respectively). The ACAs parallel one another in the midline and cannot be distinguished as 2 separate vessels. Thus, there is visualization of 3 distinct perfusion lines, the paired ACAs and each MCA, resulting in the "trident sign." There will also be a generalized blush of activity throughout the brain parenchyma, followed by visualization of the superior sagittal sinus during the venous phase.

In a positive brain-death study (Fig. 5), the radiopharmaceutical bolus goes through the common carotid arteries but stops at the base of the skull. This is because brain death results in raised intracranial pressure that exceeds the cerebral perfusion pressure supplied by the internal carotid artery. However, flow to the external carotid artery, which supplies the face and scalp, persists and may even be elevated, often leading to the "hot-nose sign" (7). It is important not to confuse hyperemic scalp injuries or facial structures with perfusion to the brain. For a positive study, there should be no visualization of the ACAs or MCAs or of the blush within the brain parenchyma. Faint activity may be seen in the superior sagittal sinus because of some drainage from superficial structures. If activity is not visualized in the carotid arteries, then an inadequate injection is suspected and the injection must be repeated.

${ }^{99} \mathrm{~m} \mathrm{Tc}-\mathrm{HMPAO}$ and ${ }^{99 \mathrm{~m}} \mathrm{Tc}-\mathrm{ECD}$ are lipophilic agents that cross the blood-brain barrier and are taken up by brain tissue in relation to regional cerebral blood flow. The recommended dose of ${ }^{99 \mathrm{~m} T c-H M P A O}$ or ${ }^{99 \mathrm{~m}} \mathrm{Tc}-\mathrm{ECD}$ is 370 1,110 MBq (10-30 mCi) (6).

Although the primary imaging using these agents consists of static views at 20 min or greater, it is also recommended that flow images are obtained during radiopharmaceutical injection as described above for ${ }^{99 \mathrm{~m}}$ Tc-DTPA (6). This will help prevent a false conclusion of brain death if there is absence of brain uptake on delayed views arising from improper preparation or instability of the radiopharmaceutical or from an infiltrated dose. The static views consist of anterior and both lateral views (Fig. 6) and a posterior view if possible. SPECT has been recommended to improve the assessment of perfusion in the posterior fossa or brain stem (8) and to help with study interpretation if significant scalp activity is present (9). However, SPECT may be difficult to perform with an unstable patient condition and the patient's associated medical equipment, and in practice this procedure is rarely used. A positive brain-death study with a brain-specific agent will demonstrate no uptake in the brain (Fig. 7).

When ${ }^{99 \mathrm{~m}} \mathrm{Tc}-\mathrm{HMPAO}$ is used, additional information on cardiac output and regional perfusion rates of organs, such as the kidneys, heart, lung, and liver, may be obtained with

FIGURE 4. Normal flow study. Series of frames from flow phase (A) and pool phase (B). There is flow up common carotid arteries (CCA) and internal carotid arteries (ICA), onward to ACAs and MCAs, and ultimately into superior sagittal sinus (SSS).

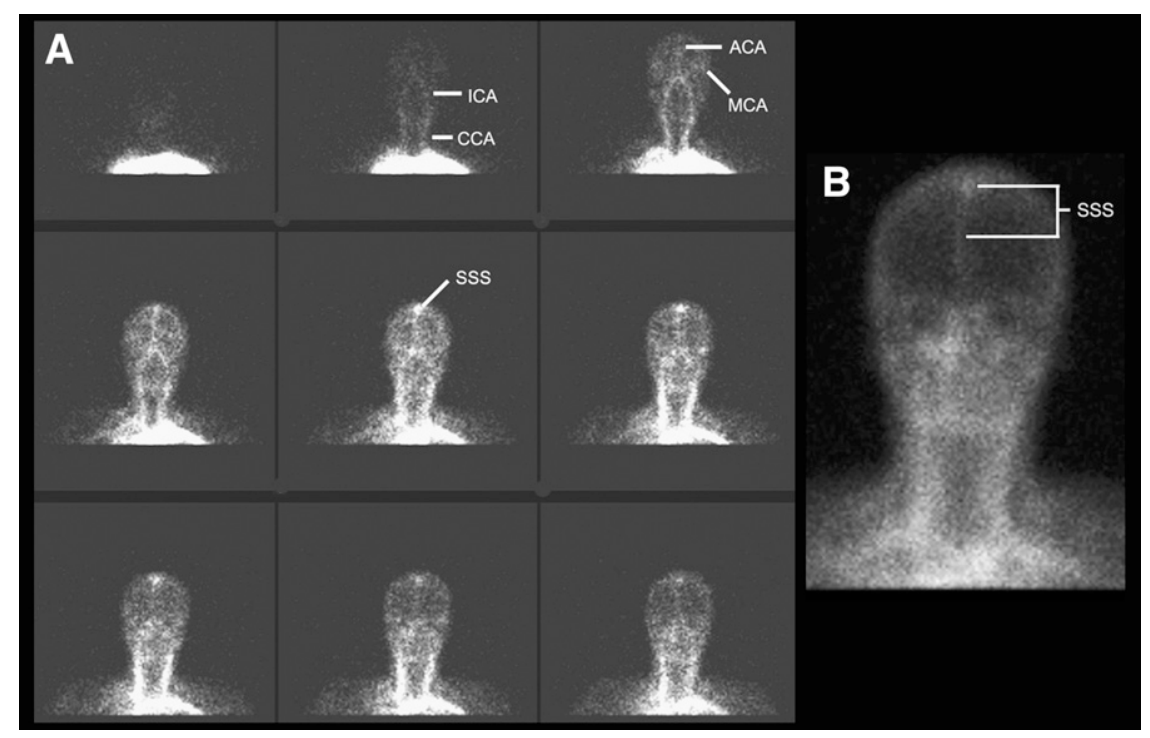




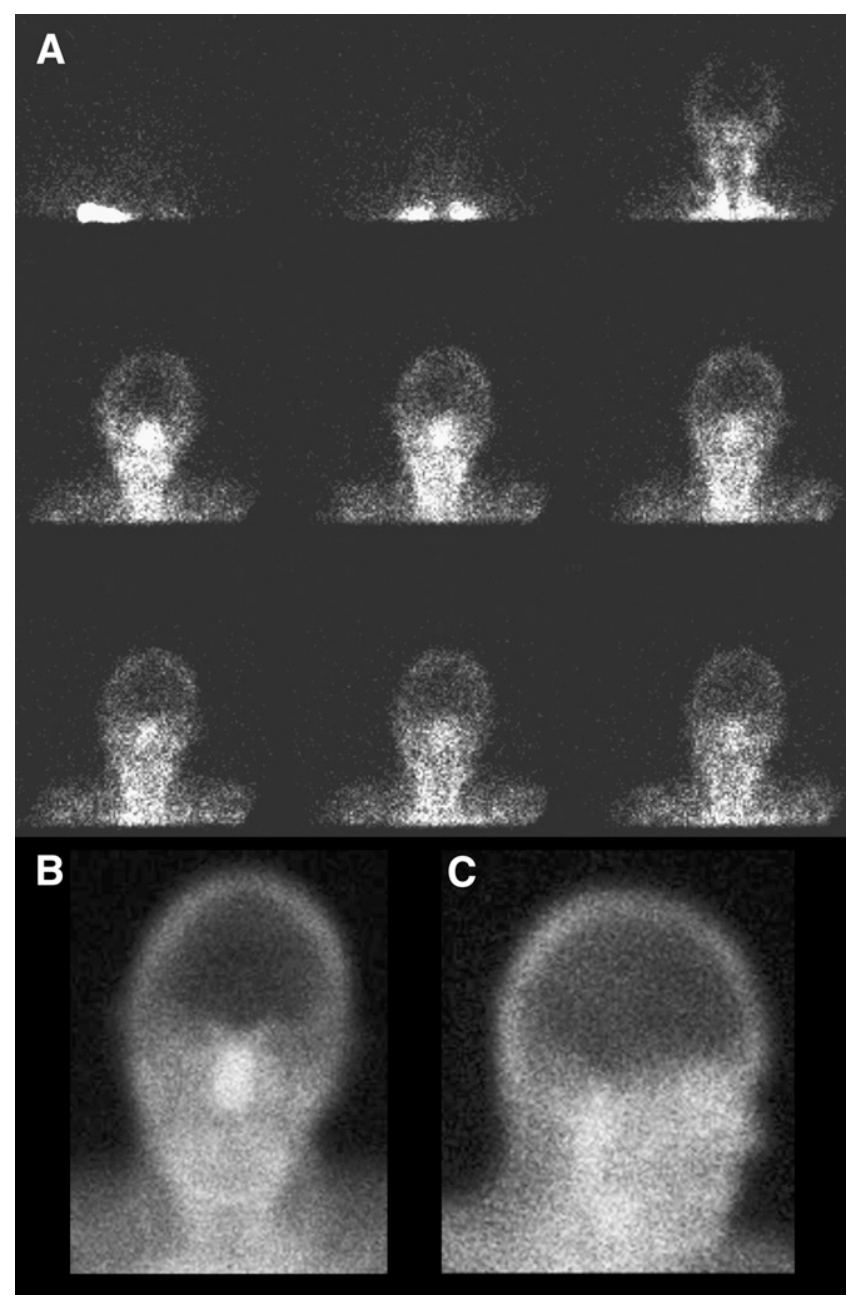

FIGURE 5. Positive brain-death study with 99mTc-DTPA. Series of images from flow phase (A) and anterior and lateral images from pool phase ( $B$ and $C$ ). There is flow up carotid arteries of neck and to scalp and facial structures (hot-nose sign), which are supplied by external carotid arteries. However, there is no flow to brain, which is supplied by internal carotid arteries.

a whole-body pass scan (8), although most institutions do not incorporate this procedure into their protocol.

If there is a small amount of perfusion to the brain or if clinically there is deterioration, it may be useful to repeat the study. The amount of cerebral uptake of ${ }^{99 \mathrm{~m}} \mathrm{Tc}-\mathrm{HMPAO}$ or ${ }^{99 \mathrm{~m}} \mathrm{Tc}-\mathrm{ECD}$ would prohibit scanning from being repeated with adequate sensitivity on the same day (10). ${ }^{99 m}$ Tc-DTPA would allow for a repeated study on the same day if necessary, whereas use of the other agents would require a delay of $24 \mathrm{~h}$ for repeated imaging.

Because ${ }^{99 \mathrm{~m}} \mathrm{Tc}-\mathrm{ECD}$ and ${ }^{99 \mathrm{~m}} \mathrm{Tc}-\mathrm{HMPAO}$ agents are brain-specific, they may be preferable to ${ }^{99 \mathrm{~m}} \mathrm{Tc}$-DTPA, in part because they are not dependent on a good bolus intravenous injection and because delayed imaging is usually definitive in assessing cerebral blood flow (6). The technologist plays an important role in the proper labeling of the brain-specific agents to prevent a false-positive study.

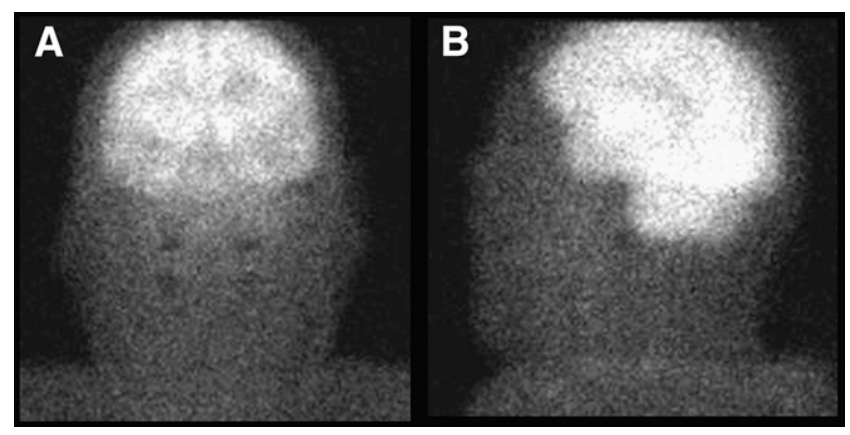

FIGURE 6. Normal ${ }^{99 m T c-E C D ~ s t u d y . ~ A n t e r i o r ~(A) ~ a n d ~ l a t e r a l ~}$ (B) views reveal normal uptake within brain.

Quality control should be performed as outlined in the package insert (11), and proper labeling should be verified before patient administration.

An alternative ancillary imaging test is cerebral angiography, in which a catheter is introduced via a femoral artery and advanced to the carotid arteries, and radiographic contrast is injected. As with the nuclear medicine study, the premise is that, because of increased intracerebral pressures in brain death, no flow will be seen to the brain.

Angiography is more invasive than scintigraphy. The radiographic contrast can cause idiosyncratic reactions and can result in renal dysfunction, which may be relevant with respect to organ donation. Transcranial doppler ultrasound and MRI-based angiography both show promise but have not been sufficiently validated (4).

\section{TESTICULAR SCANNING}

The testicles are responsible for production of sperm and testosterone. The testicles develop in the retroperitoneum of the abdomen and descend in utero to exit the pelvis and reside in the scrotum. The epididymis is a small structure on the posterior aspect of the testicle, responsible for storing sperm. The nuclear medicine testicular scan is used in the setting of acute scrotal pain to differentiate between a variety of entities, primarily testicular torsion and orchitis or epididymitis.

When the testicles descend into the scrotum, a portion of the peritoneal lining descends with them and covers a portion of the testicles. Where not covered by this layer (the tunica vaginalis), the testicles and epididymis are anchored to the scrotum. If the tunica vaginalis covers too much of the testicle, it may be inadequately anchored to the scrotum, allowing it to rotate within the scrotum. This deformity, known as the "bellclapper deformity," is implicated in most cases of testicular torsion (12). The peak age of presentation is around puberty; this entity is rare after the age of $30 \mathrm{y}$. Torsion results in compromise of blood flow to the testicles, either through direct arterial compression or decreased supply secondary to obstruction of the venous outflow, resulting in ischemia and potentially infarction. Patients present with acute scrotal pain. 


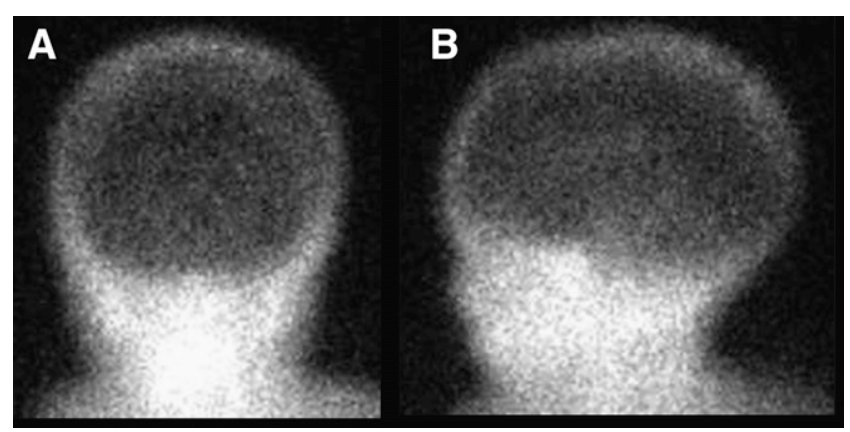

FIGURE 7. Positive brain-death study with ${ }^{99 m T c-E C D . ~ A n t e-~}$ rior $(A)$ and lateral $(B)$ views reveal no uptake within the brain in a child.

Emergency surgery is required to untwist the testicle to prevent necrosis; the salvage rate tends to reflect the time passed between onset of the event and the surgical detorsion. In one study (13), the salvage rate was $100 \%$ when surgery occurred within $6 \mathrm{~h}, 70 \%$ for surgery between 6 and $12 \mathrm{~h}$, and only $20 \%$ when surgery occurred more than $12 \mathrm{~h}$ after onset of symptoms. Consequently, a request for a testicular scan in the setting of acute scrotal pain must be treated as an emergency. In fact, when suspicion of torsion is high, it is generally recommended $(12,14)$ that the patient be taken directly to surgery without imaging. In addition to untwisting the involved testicle, both testicles are anchored to the scrotum at the time of surgery to prevent future events.

The main entity to be distinguished from torsion in the setting of acute scrotal pain is inflammation. Most commonly this involves only the epididymis (epididymitis), but sometimes the testicle may be involved (orchitis). Most commonly, the etiology is nonspecific, but specific causes may include gonorrhea, syphilis, tuberculosis, or mumps. The presentation is often more gradual than with torsion; patients are more likely to have fever, but there is considerable overlap and imaging is often required, particularly given the significance of missing a case of torsion.

The testicular scan is predicated on assessing the blood flow to the scrotum and contents. No specific patient preparation is required for this study. Privacy should be observed and, depending on departmental or institutional policy, an escort may be required. After a clear and detailed interview is conducted and an explanation of the scan is given, the patient is asked to lie supine on the imaging table, with his legs abducted and feet together in a frog-leg position to decrease background activity from the thighs. The penis is directed in a cranial direction and may be taped in place to avoid overlap with the scrotum. A towel may be draped under the scrotum and over the thighs, acting as a sling to raise the scrotum. Once proper positioning is obtained, a sheet, towel, or light blanket should be used for the modesty of the patient. Proper positioning is vital to a proper diagnosis, and with detailed instruction the patient is usually more than willing to assist.
A camera with a low-energy all-purpose collimator should be placed parallel and close to the patient, to minimize the distance between the scrotum and the camera. A pinhole collimator may be used with the pediatric population. ${ }^{99 \mathrm{~m} T c}$-pertechnetate $(555 \mathrm{MBq}[15 \mathrm{mCi}]$, with a minimum of $185 \mathrm{MBq}$ [5 $\mathrm{mCi}$ ] for the pediatric population) is injected intravenously, with the acquisition started immediately to ensure the flow portion of the study is not missed. This includes dynamic imaging at $2-5 \mathrm{~s} /$ frame for 1-2 min of the anterior pelvis, with further static imaging after the flow portion for at least 500,000 counts $(500 \mathrm{~K})$ per static image.

Real-time anatomic correlation by the nuclear medicine physician may be required, such as by placing a hot source over the raphe of the scrotum or over the testicle itself (Fig. 8). Physical examination of the scrotum may be required to exclude, for example, a hydrocele (fluid collection) or hernia as a false-positive cause of a photopenic defect.

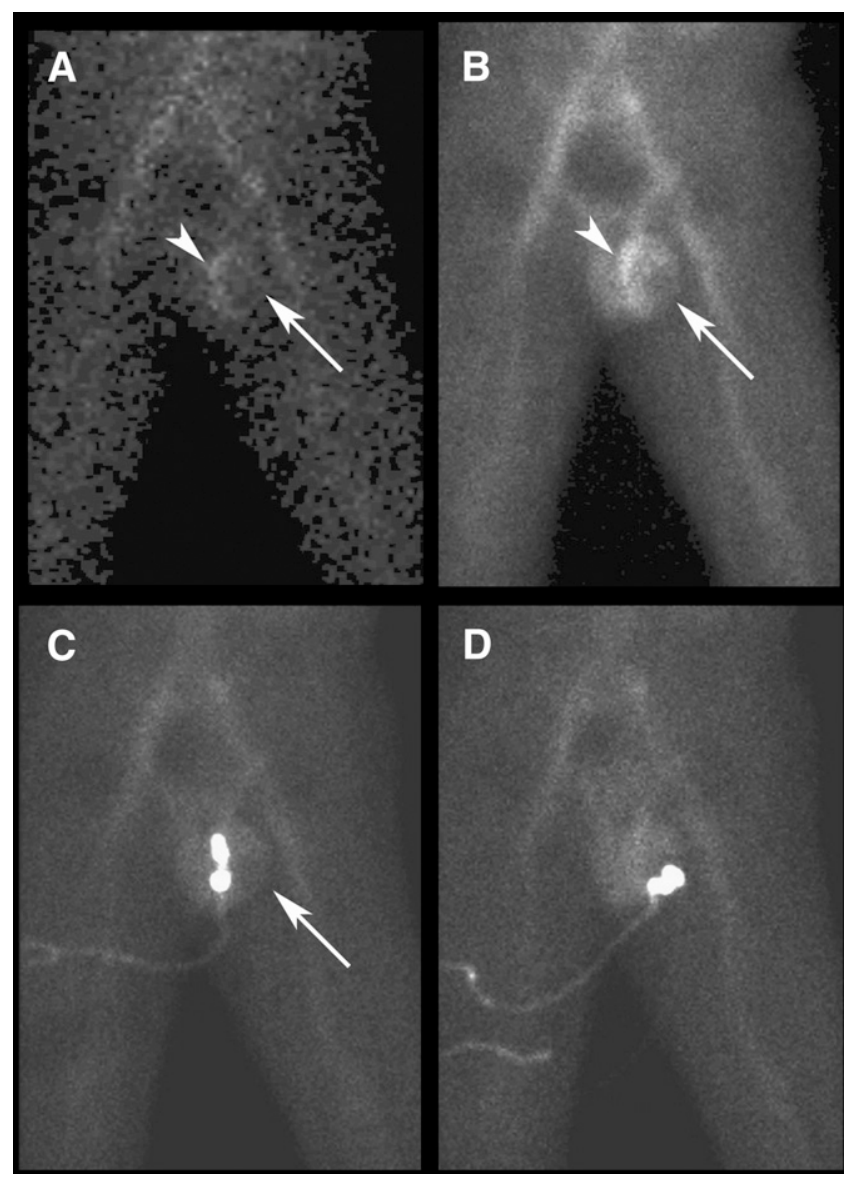

FIGURE 8. Testicular torsion. Single frame from flow sequence $(A)$ and pool image (B) from testicular scan. On both sequences, there is no flow to left testicle (arrows). Rim of increased flow superior and medial to testicle (arrowhead) results from reactive hyperemia ("rim sign"). To confirm that absence of perfusion truly corresponded to left testicle, hot $99 \mathrm{~m} T \mathrm{c}$ source was placed sequentially over raphe between left and right sides of scrotum (C) and directly over left testicle itself (D) under palpation by the nuclear medicine physician. 
False-negative scan results have occurred in the setting of extremely late torsion and with small or retracted scrotums. Still, the overall accuracy is impressive at 95\% (12).

In the normal testicular scan, blood flow is demonstrated in the iliac and femoral arteries and the penis and symmetrically and faintly in the scrotum and testicles. The findings in the setting of torsion depend to some extent on the timing since the onset of the event, but the overriding principle is that there is decreased perfusion to the testicle. Increased flow may be seen in the surrounding scrotum as a result of hyperemia (the "rim sign"). Abrupt cutoff of the iliac artery supplying the testicle is occasionally seen (the "nubbin sign"). If the testicle spontaneously untwists before imaging, there may actually be increased flow to the testicle due to hyperemia.

The hallmark in inflammation or infection is increased blood flow. On the pool images, this may be seen to be lateral within the scrotum in epididymitis or medial in orchitis. In all cases, the interpreting physician must be aware of the clinical presentation, including which side is painful, as asymmetry may be due to abnormally increased uptake on one side, consistent with inflammation, or abnormally decreased uptake on the other side, consistent with torsion.

Several other entities may be encountered on the scan. In addition to epididymitis and orchitis, a frank abscess may be present in the scrotum. As expected, this will result in increased uptake. In addition to torsion of the entire testicle, torsion of one of several small appendages may occur, for example the testicular appendage or appendix epididymis. Imaging in torsion of these small appendages is often normal, although a small area of photopenia may be appreciated. Testicular tumors are rarely a cause of acute scrotal pain and hence rarely lead to a nuclear medicine testicular scan. However, a tumor may be encountered incidentally. The appearance can be variable, depending on the relative vascularity, but usually there would be increased flow on the initial phase.

Besides scintigraphy, the acute scrotum may be evaluated using ultrasound. The differentiation between torsion and inflammation relies on demonstrating decreased or increased blood flow using color doppler, analogous to the nuclear medicine approach (Figs. 9 and 10). The reported accuracy of scintigraphy (95\%) exceeds that of ultrasound (88\%) (12). The ultrasound examination may be limited by pain, as direct contact with the scrotum is required. Given the urgent need for assessment, the choice of management may be dictated by the more ready availability of ultrasound than of nuclear medicine after hours in some institutions.

\section{QUANTITATIVE LUNG PERFUSION STUDY}

Lung cancer is the leading cause of cancer death in both men and women. In early stages, patients with lung cancer may be candidates for surgical resection of the tumor in an attempt at cure. Depending on the size and location of the tumor, surgery may consist of lobectomy (partial or total) or pneumonectomy. Before a surgery of this nature, pulmonary function must be evaluated to ensure that sufficient function will remain after the operation. This assessment is particularly relevant, as patients with lung cancer often have decreased preoperative lung function due to the common risk factors of age and smoking. Standard work-up includes pulmonary function tests assessing forced expiratory volume in $1 \mathrm{~s} \mathrm{(FEV1)} \mathrm{and} \mathrm{diffusion} \mathrm{capacity} \mathrm{for} \mathrm{carbon} \mathrm{monoxide.}$ FEV1 may be expressed as an absolute value (L) or as a percentage of predicted value based on age, height, sex, and race. Patients whose preoperative FEV1 is greater than $2 \mathrm{~L}$ or more than $80 \%$ predicted are considered candidates for pneumonectomy, and those whose FEV1 is greater than 1.5 $\mathrm{L}$ are considered candidates for lobectomy, without further pulmonary evaluation (15).

However, when these criteria are not met, further assessment is indicated to predict postoperative function, typically with a quantitative perfusion study. Several studies have demonstrated a high risk for perioperative death and pulmonary complications when the predicted postoperative FEV1 is less than $40 \%$ or diffusion capacity for carbon monoxide is less than $40 \%$ (15).

Quantitative perfusion lung studies are simple to perform, and results may be obtained almost immediately after the study is performed. No specific patient preparation is necessary. The radiopharmaceutical used is ${ }^{99 \mathrm{~m}} \mathrm{Tc}-$ macroaggregated albumin $\left({ }^{99 \mathrm{~m}} \mathrm{Tc}-\mathrm{MAA}\right)$, with a recommended dose range of 37-148 MBq (1-4 mCi) (16). Quality control of both radiochemical purity and particle size should be performed and analyzed as outlined in the product monograph (17). The recommended number of particles for intravenous injection is in the range of 200,000-700,000. The number of particles should be lowered for pediatric

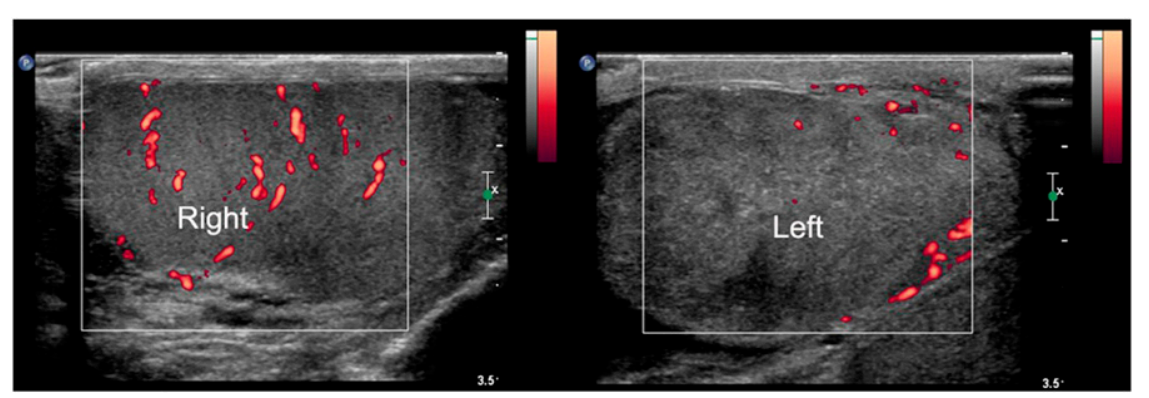

FIGURE 9. Doppler ultrasound images longitudinally through each testicle on same day as nuclear medicine scan in Figure 8. There is decreased blood flow (color) to left testicle relative to right. 


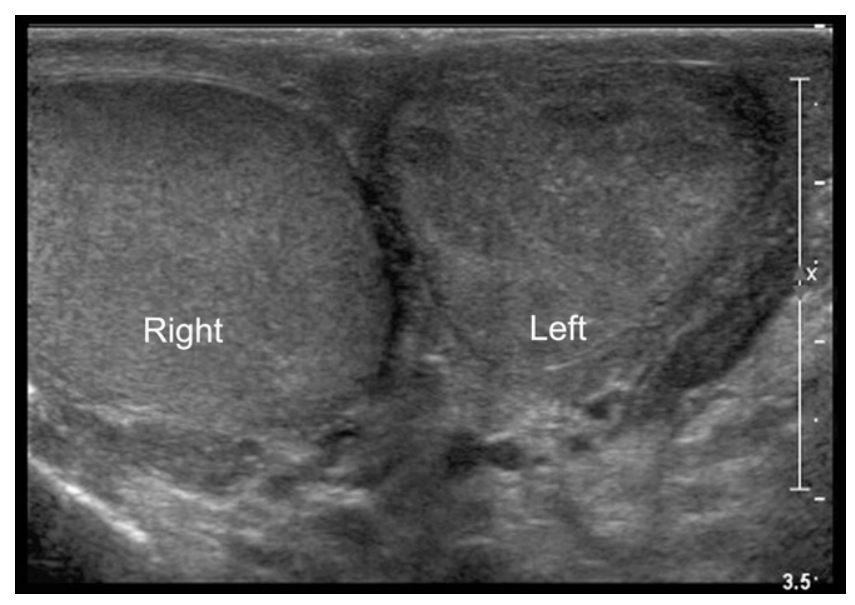

FIGURE 10. For patient in Figure 9, transverse gray-scale images from follow-up ultrasound 3 wk later. Marked heterogeneity of left testicle is due to infarction. Right testicle demonstrates normal homogeneous echotexture.

patients, those patients with right-to-left shunts, and those patients with severe pulmonary hypertension (16). It is important to agitate the vial before dose drawing, because the ${ }^{99 m}$ Tc-MAA particles will settle to the bottom of the vial. The syringe of ${ }^{99 \mathrm{~m}} \mathrm{Tc}-\mathrm{MAA}$ should also be shaken before patient injection, especially if the syringe is not used immediately after dose draw from the vial. Shaking will prevent the ${ }^{99 \mathrm{~m}} \mathrm{Tc}-\mathrm{MAA}$ particles from sticking together in the syringe. The patient is injected intravenously while in the supine position to ensure homogeneous distribution of the radiopharmaceutical in the lungs. Blood should not be drawn into the syringe during injection because of the possibility of clumping of the ${ }^{99 \mathrm{~m}} \mathrm{Tc}-\mathrm{MAA}$ in the lungs. Static images are acquired in both the anterior and posterior chest positions, typically for $500 \mathrm{~K}$.

With the aid of computer software, ROIs are placed over the right and left lungs. Each lung is then further divided into thirds to create superior, middle, and inferior ROIs. The geometric mean of the anterior and posterior counts in each region is calculated, and the percentages for each region and each lung are calculated. The geometric mean-the square root of the product of the anterior and posterior counts-is frequently used in nuclear medicine to estimate the counts in an organ based on anterior and posterior planar imaging, is considered more representative than the familiar arithmetic mean (anterior plus posterior counts divided by 2), and is commonly used for dosimetry assessments as well. The division of the lungs into superior, middle, and inferior regions does not exactly correlate with the anatomic divisions into lobes but is reasonably representative and allows estimation of postoperative lung function after lobectomy. On the other hand, in assessment before pneumonectomy, this technique accurately demonstrates the preoperative pulmonary contribution of each lung.

The predicted postoperative pulmonary function is then estimated by applying the results of the quantitative perfusion study to those of the preoperative pulmonary function tests. For example, in the patient in Figure 11, removal of the left lung is being planned. The nuclear medicine study has determined that this lung contributes $30 \%$ of the total pulmonary function. Hence it can be estimated that the planned left pneumonectomy will reduce the preoperative FEV1 by $30 \%$ (Fig. 12).

Some authors have advocated quantitative ventilation imaging (18) rather than perfusion imaging in this scenario, but most advocate using perfusion imaging. Quantitative assessment based on ventilation could be particularly inaccurate when using aerosolized ${ }^{99 \mathrm{~m}} \mathrm{Tc}$ agents, especially in the setting of obstructive airway disease. In this setting, patients with obstructive airways may show central airway deposition of the radiopharmaceutical and, therefore, the concentration of the radiopharmaceutical in the lungs will not accurately correlate with the patient's pulmonary function.

In addition to preoperative assessment for lung cancer, there are other applications of the quantitative lung scan. Rarely the study may be requested in advance of radiation therapy to the lungs in patients with borderline pulmonary function. It may also be indicated in patients undergoing lung reduction surgery, a procedure that removes the superior portions of a lung or lungs because of the presence of bullae in patients with emphysema. We perform this study not uncommonly in the pediatric population to assess relative lung function in children with congenital heart disease including stenosis of a pulmonary artery. The study may be used to establish when an intervention such as dilatation of the pulmonary artery is indicated and may be subsequently used to evaluate the effectiveness of the therapy through comparison with the preintervention study.

The predicted postoperative lung function may also be estimated by multiplying the preoperative function by the ratio of lung segments being removed to the total number of lung segments (18 segments). However, this approach is less accurate than the approach using quantitative perfusion, as it assumes all segments are of the same size and pulmonary function is homogeneous throughout the lungs, a situation that is not typical in this patient population.

\section{LYMPHOSCINTIGRAPHY}

In recent years the use of nuclear medicine to assess the lymphatic system has become well known through the

FIGURE 11. Chest radiograph in a patient with lung cancer. Large tumor is seen in left lung.

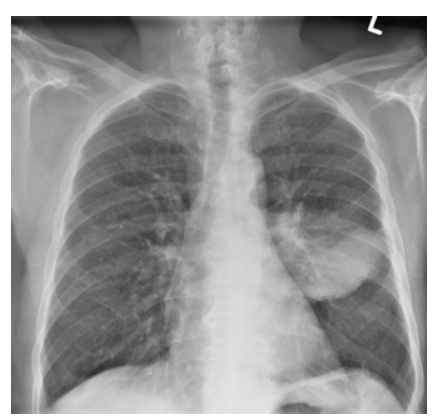




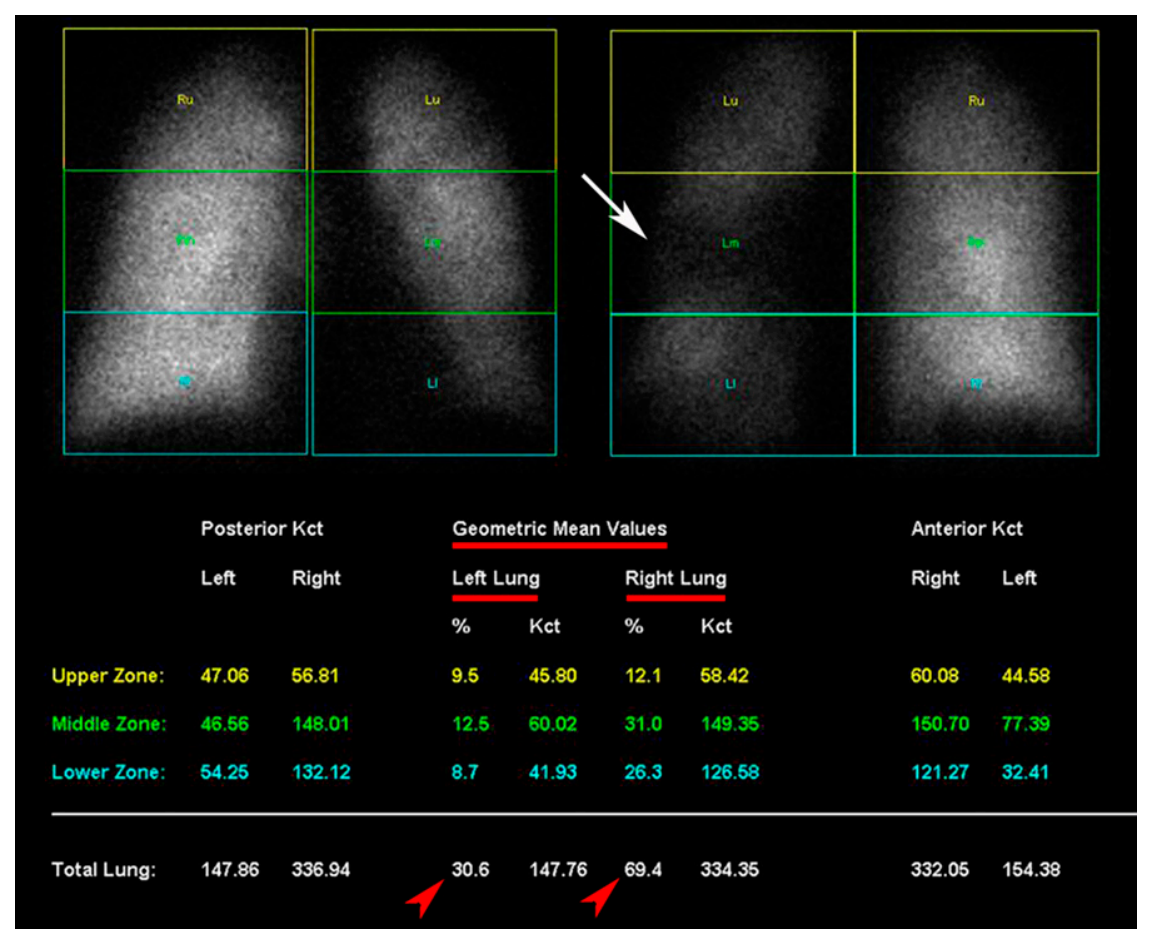

FIGURE 12. Quantitative perfusion scan in same patient as in Figure 11. Large perfusion defect from tumor is appreciated on posterior image (arrow). Analysis reveals that left lung contributes $30.6 \%$ and right lung $69.4 \%$ of total pulmonary function (arrowheads). Hence, it is anticipated that removal of left lung will result in decrease of approximately $30 \%$ of total pulmonary function. widespread use of sentinel node studies. However, lymphoscintigraphy has been used for many years in other settings, and it is these less familiar roles that are reviewed here.

The lymphatic system plays a role in the immune (lymph nodes, spleen, and other tissues) and circulatory systems. In this latter role, the lymphatic system is responsible for transporting cellular debris, metabolic waste products, and fluid excesses from the interstitial tissues back to the venous system (19). In the extremities, there are parallel deep and superficial lymphatic channels that generally parallel the venous system. The deep and superficial systems of the legs converge in the pelvis, and those of the arms converge in the axillae. Drainage is central, and ultimately the lymphatic system drains into the venous system via the thoracic duct, the main lymphatic conduit in the chest, at the junctions of the jugular and subclavian veins.

Scintigraphic lymphatic mapping is used primarily in the setting of lymphedema, swelling of an extremity as a result of lymphatic malformation or malfunction. Lymphedema may be classified as primary (present at birth although not evident until later in life) or secondary (acquired as a result of infection, inflammation, trauma, or neoplasm or in association with venous obstruction). Worldwide, the most common etiology is parasitic infection, often filariasis. In the Western world, lymphedema is often associated with cancer, either through direct invasion or obstruction by tumor, or as a result of surgical or radiation therapy. For example, lymphedema can occur in the upper extremity after axillary node dissection for breast cancer, resulting in significant morbidity. Of note, nuclear medicine has played a role in reducing this complication through the use of the less invasive sentinel node concept (20).

Lymphoscintigraphy is a safe, minimally invasive, repeatable method of assessing for lymphedema. Contrast lymphangiography has been used in the past. This method, which involves tedious cannulation of the peripheral lymphatics and injection of radiographic contrast, is difficult and time-consuming and nonphysiologic, as the tracer is injected directly into the lymphatics under some pressure (in lymphoscintigraphy the injection is into the dermal tissues, and subsequent uptake by the lymphatics is under physiologic conditions). In addition, reactions to the contrast medium have occurred.

A variety of different radiopharmaceuticals have been used in lymphoscintigraphy, including ${ }^{198} \mathrm{Au}$ (historic),

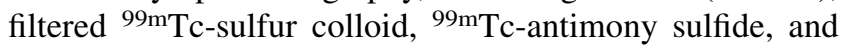
${ }^{99 m}$ Tc-nano and -microaggregated albumin (19). In North America, filtered ${ }^{99 \mathrm{~m}} \mathrm{Tc}$-sulfur colloid is predominantly used. Dermal injections of tracer are made in the second and fourth web spaces bilaterally. That is, both hands are injected and imaged when lymphedema is suspected in the upper extremities, and both feet are injected when evaluating the lower extremities.

Because the dermal space is tight, a small volume, and hence high specific activity, is required (e.g., 9.25 MBq/0.1 $\mathrm{mL}[250 \mu \mathrm{Ci} / 0.1 \mathrm{~mL}])$.

The dose for adults is generally $37 \mathrm{MBq}(1 \mathrm{mCi})$ total. Therefore, imaging of the legs should require approximately $9.25 \mathrm{MBq}(250 \mu \mathrm{Ci})$ per injection (2 injections per extremity). One may choose to withdraw $0.05 \mathrm{~mL}$ of air or saline before withdrawing the sulfur colloid, to ensure the patient receives as much of the dose as possible. For 
children, 13-18.5 MBq (350-500 $\mu \mathrm{Ci})$ total may be used. If the patient wears elastic stockings for lymphedema, then the stockings should be removed at least 3-4 $\mathrm{h}$ before the study (2). If the patient is being evaluated for upper-limb lymphedema, hand-grip exercises may be used to enhance lymphatic absorption. Similarly, for lower-leg evaluation, instructing the patient to walk or climb stairs after injection will enhance lymphatic absorption.

After this brief, low-level exercise, imaging is initiated. Imaging begins just proximal to the injection site, as most of the injection (up to 95\%) will not be absorbed by the lymphatics and intense activity will remain at the sites of injection. Imaging subsequently consists of a series of sweeps moving progressively more proximal as the tracer migrates centrally through the lymphatics. Once the radiopharmaceutical has migrated to the junction of the lymphatic and venous systems in the upper thorax, it will circulate in the venous system and become trapped in the liver and spleen (as when injected intravenously in a liverspleen study, though much fainter), so visualization of the liver indicates completion of migration through the lymphatic system and is usually taken as the endpoint of the study. A ${ }^{57}$ Co sheet source may be used as a transmission source to outline the patient's body for visual enhancement.

Findings in the setting of lymphedema (Fig. 13) include delayed transit of activity relative to the normal side, less activity in the lymphatic channels, and less activity in lymph nodes. A common finding is dermal backflow, in which activity is shunted from the abnormal deep lymphatics to the superficial lymphatics, resulting in uptake in a fine mesh of superficial lymphatics over the distal aspect of the lower legs ("stocking sign").

A less common indication for lymphoscintigraphy is evaluation of lymphatic leaks. Figures 14 and 15 are images of a patient who had recently undergone right lung surgery for excision of metastases. A pleural effusion developed after the operation, and it was suspected that this may have arisen from damage to the thoracic duct, resulting in leakage of lymphatic fluid into the pleural space (chylothorax). In this study, dermal injection of filtered ${ }^{99 \mathrm{~m}} \mathrm{Tc}$-sulfur colloid in the feet resulted in migration through the lymphatic system and spillage into the right pleural space, confirming that the pleural effusion was due to disruption of the thoracic duct.

\section{SALIVARY GLAND SCINTIGRAPHY}

The salivary glands are exocrine glands that surround the oral cavity and secrete saliva, which plays an important role in the digestion of food and in oral hygiene. There are 3 major pairs of salivary glands: parotid, submandibular, and sublingual. The parotid glands are anterior and inferior to the ear. They secrete saliva into the oral cavity via Stensen ducts. The submandibular glands are on the floor of the mouth and secrete saliva via Warthin ducts. The sublingual glands are on the floor of the mouth, lateral to the tongue, and secrete saliva via sublingual or Warthin ducts.

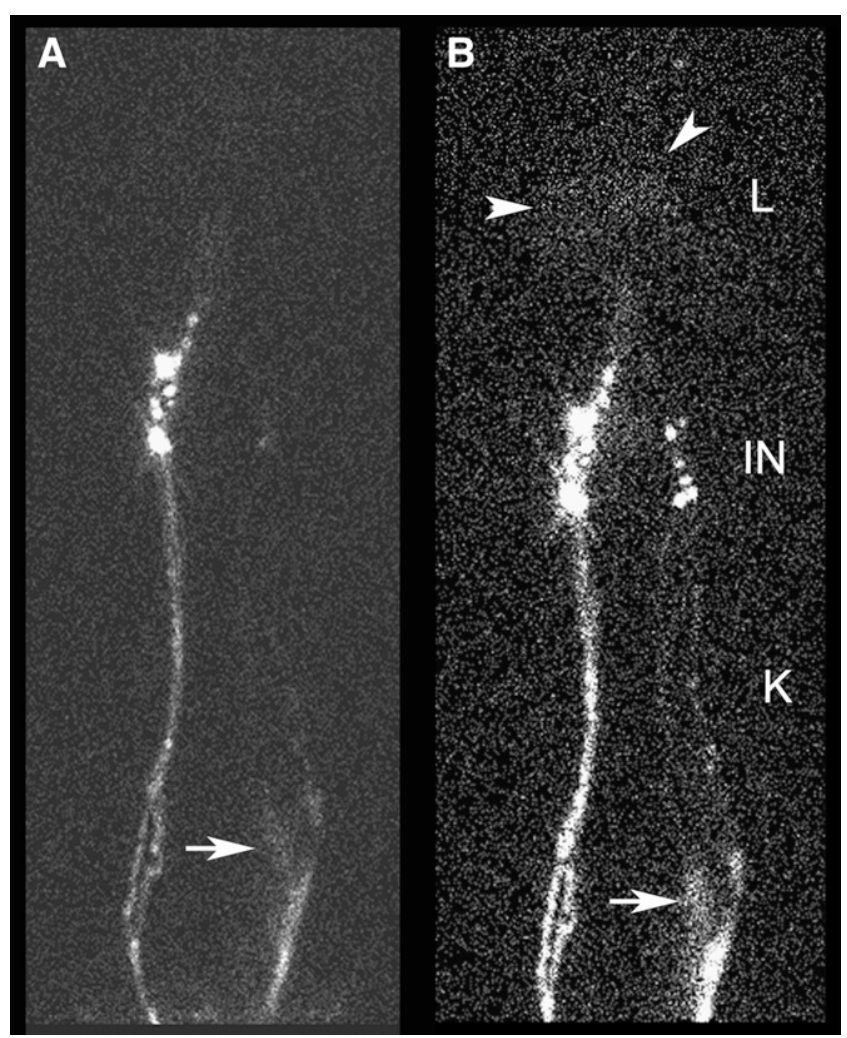

FIGURE 13. Lower-extremity lymphedema. Anterior images from upper abdomen to ankles at $1 \mathrm{~h}(\mathrm{~A})$ and $3 \mathrm{~h}(\mathrm{~B})$. Right side is normal. Colloid ascent on left is delayed and of lower volume, and activity is much lower in left-sided lymph nodes. In lower left leg there is shunting to superficial lymphatics (arrow). Faint activity is seen in liver (arrowheads), indicating endpoint of study. $\mathrm{L}=$ level of liver; IN = level of inguinal nodes; $\mathrm{K}=$ level of knees.

Salivary gland scintigraphy may be used to assess the function of the salivary glands, to evaluate salivary duct patency, or to help characterize tumors. Imaging the salivary glands with pertechnetate is easily attainable because of the ability of the glands to transport the pertechnetate anions from the surrounding blood supply into the salivary glands.

The parotid and submandibular glands are clearly visualized, but the normal sublingual glands are not routinely seen. Patients should not be given thyroid-blocking agents, such as iodide or perchlorate, within $48 \mathrm{~h}$ before the scan

FIGURE 14. Postoperative chest radiograph. Large right pleural effusion is seen (arrowheads). Chest drainage tube is in place (arrow).

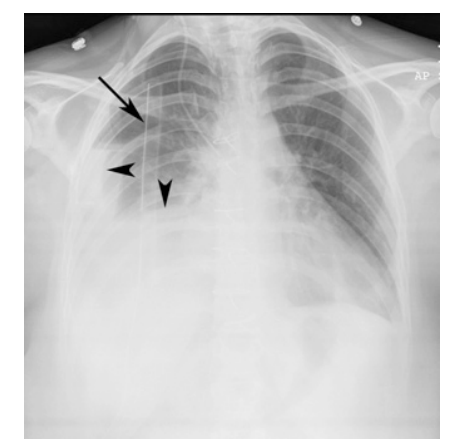




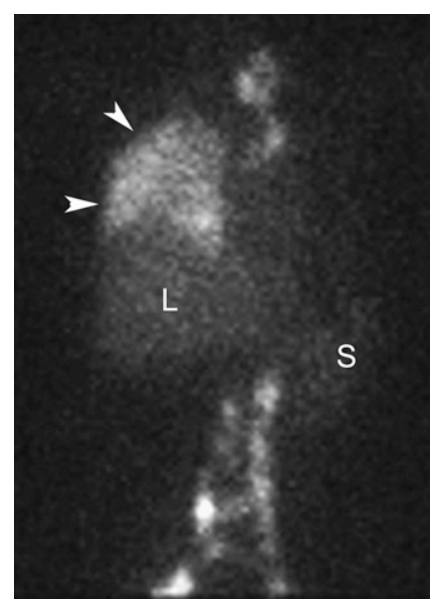

FIGURE 15. Anterior image from lymphoscintigraphy study in same patient as in Figure 14, extending from neck to upper pelvis. Abnormal accumulation of radiopharmaceutical is seen throughout right side of thorax (arrowheads), confirming lymphatic leak. Appropriate uptake is seen in abdominal and superior mediastinal lymphatics and within liver (L) and spleen (S).

(2). The patient is asked to lie supine in the Water's position (chin and nose touching collimator face), and approximately $555 \mathrm{MBq}(15 \mathrm{mCi})$ of pertechnetate are administered intravenously. A dynamic blood flow study is performed immediately after intravenous injection for 3-5 s/frame for 1-2 min, followed by $20 \mathrm{~min}$ of dynamic imaging for 2-3 $\mathrm{min} /$ frame to assess function of the glands (21). Right and left lateral head images may then be taken for $500 \mathrm{~K}$. Trapping of the pertechnetate should occur within 1 min of intravenous injection in normal glands. Peak activity occurs in the glands within 10 (22) to $21 \mathrm{~min}$ (23).

Once the first dynamic study is complete, the functional (excretory) aspect of the salivary glands is assessed with a second dynamic study after oral administration of lemon juice (lemon stimulation). Ensure that the patient has no allergies to lemon juice. This scan is obtained with the patient positioned as in the prestimulus dynamic acquisition for 2-3 $\mathrm{min} /$ frame for $20 \mathrm{~min}$. Once the acquisition has started, the patient is asked to drink a mouthful of lemon juice via a straw and hold this in the oral cavity for a few seconds before swallowing and then continue to hold as still as possible for the remainder of the acquisition. Static images in the right lateral and left lateral positions are acquired as needed. Alternatively, a single longer dynamic acquisition may also be used, incorporating both the prestimulus and poststimulus phase. For example, one may choose to set up the dynamic acquisition for 40 min and administer the lemon juice via a straw to the supine patient 20 min into the dynamic acquisition. It is important to keep the patient unaware of the lemon juice stimulation until the time of administration, to prohibit psychologic stimulation of the glands. Normal glands should exhibit a significant decrease in activity almost immediately after lemon stimulation, with a resultant increase in activity in the oral cavity (Fig. 16).

During the prestimulus phase, an increase in uptake may be due to local inflammation or infection. For example, acute sialadenitis on scintigraphy displays an increase in blood flow, resulting in marked uptake and retention of pertechnetate within the entire gland. On the other hand, in chronic sialadenitis uptake is variable depending on the stage, eventually leading to a complete loss of uptake of pertechnetate in the affected glands. Decreased uptake and decreased excretion of the pertechnetate from the salivary glands may occur in patients with Sjogren syndrome, an autoimmune disorder that affects exocrine glands such as lacrimal and salivary glands. However, it may be difficult to discern whether these abnormalities are due to Sjogren syndrome or other causes of chronic inflammation. One clue is that Sjogren syndrome will often affect all of the salivary glands, whereas most other causes of inflammation will typically be more focal. Radiation treatment to the head and neck or ${ }^{131}$ I thyroid therapy for thyroid cancer may also cause functional impairment of the salivary glands.

Salivary duct obstruction may be caused by food, foreign bodies, mucus plugs, tumors, cysts, surgery, or trauma. The obstruction may cause painful swelling in the patient,

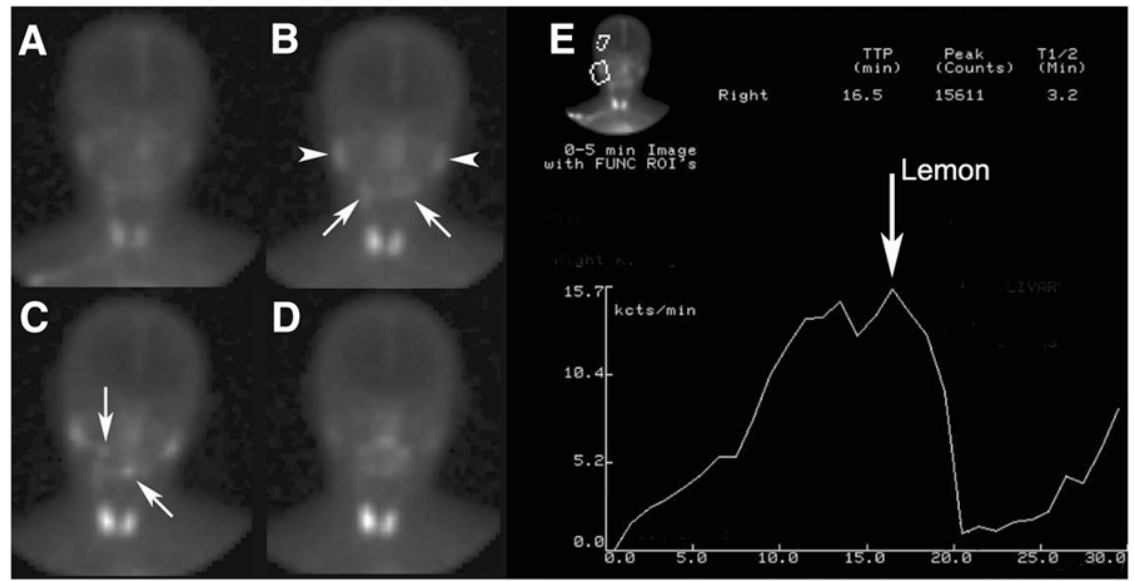

FIGURE 16. Normal salivary gland function with $99 \mathrm{~m} T c-$ pertechnetate. Images were obtained at 4 time points during study (A-D). Shortly after injection (A), marked uptake in thyroid and milder uptake in salivary glands is seen. Fifteen minutes later (B), uptake has further increased in parotid glands (arrowheads) and submandibular glands (arrows). Immediately after lemon juice stimulation (C), activity is visualized in oral cavity (arrows). Three minutes later (D), 99mTcpertechnetate has largely left salivary glands and is seen in oral cavity. (E) Time-activity curve. First ROI has been placed over right parotid gland (upper left of image) and activity has been plotted as function of time. Second ROI has been placed over scalp for purposes of background subtraction. Curve demonstrates normal uptake by gland and appropriate rapid emptying in response to lemon juice stimulation. Similar analyses were performed of other 3 major salivary glands. 
especially when salivary flow rates are high. On the prestimulus phase, uptake may be increased (secondary to resultant inflammation), normal, or decreased (secondary to dysfunction arising from the obstruction). However, the hallmark in obstruction is poor or absent clearance of activity after lemon stimulus. The dilated duct involved may or may not be seen on imaging.

For analysis purposes, ROIs may be drawn around the salivary glands to produce time-activity curves. A variety of semiquantitative parameters have been applied to these curves to assess degree of uptake into the glands, peak accumulation, and clearance of activity (Fig. 16). Studies have shown that use of salivary gland imaging, along with such semiquantitative analysis, provides a means of obtaining a reproducible tool for diagnosis and follow-up of salivary function (24). This may also be helpful in assessing therapeutic response. Alternatively, simple qualitative analysis may be performed by observation of the dynamic acquisition. If one is comparing uptake in relation to the thyroid gland, normal thyroid function is assumed.

A second, less common, form of salivary gland scintigraphy is the ${ }^{99 \mathrm{~m}} \mathrm{Tc}$-pertechnetate scan to aid in the differentiation of salivary gland masses. Primary tumors of the salivary glands include malignant tumors such as mucoepidermoid and adenoid cystic tumors and benign lesions such as Warthin tumors or pleomorphic adenomas. Secondary tumors include lymphoma and metastases. Salivary gland tumors are primarily imaged with CT or MRI, and definitive diagnosis requires biopsy. However, Warthin tumors possess the relatively unique property of concentrating ${ }^{99 \mathrm{~m}} \mathrm{Tc}$-pertechnetate and retaining it after lemon stimulation (Figs. 17 and 18). Hence if a Warthin tumor is suspected, a ${ }^{99 \mathrm{~m}} \mathrm{Tc}$-pertechnetate scan may be requested. If the scan results are positive, surgery can be foregone, as Warthin tumors are benign. Uptake in a salivary gland mass is not completely specific for Warthin tumors, as similar findings have been reported in some other benign tumors, including lymphoepithelial cysts, pleomorphic adenomas, and oncocytomas (25).

Scintigraphy is the only imaging modality that assesses salivary gland function. In the setting of obstruction, radiographic sialography may be performed. This procedure involves cannulation of the duct exit in the mouth and injection of radiographic contrast. This study may be

FIGURE 17. Patient with palpable left neck mass. CT scan through neck reveals heterogeneous mass (arrow) arising from left parotid gland. Arrowheads indicate parotid glands.

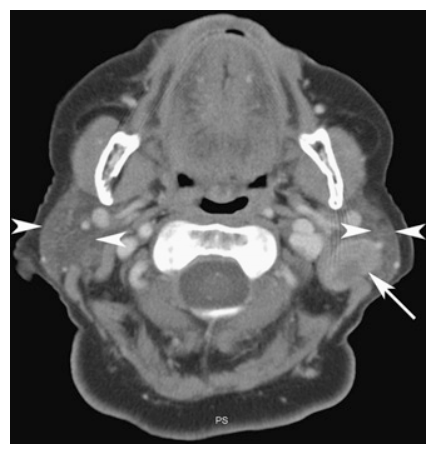

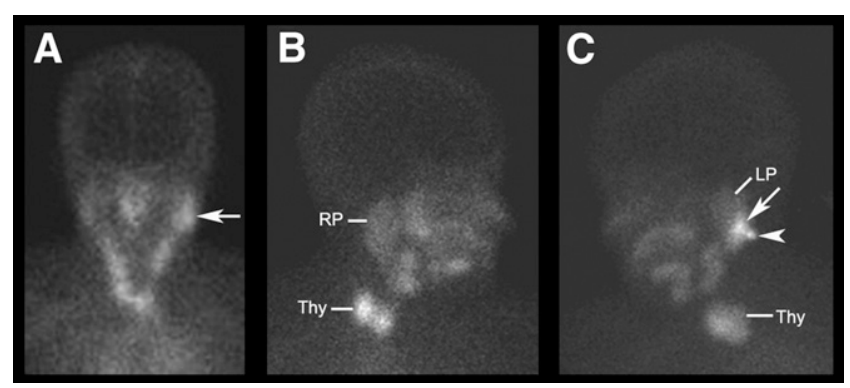

FIGURE 18. Anterior (A), right lateral (B), and left lateral (C) images from ${ }^{99 m T c-p e r t e c h n e t a t e ~ s t u d y ~ o f ~ p a t i e n t ~ i n ~ F i g u r e ~} 17$. Focus of increased uptake (arrows) in left parotid gland (LP) correlates with mass on CT scan. Hot ${ }^{99 m T c}$ marker has been placed on skin over palpable mass (arrowhead), confirming that increased uptake is in mass. Uptake is normal throughout all other salivary glands and thyroid gland (Thy). RP = right parotid gland.

uncomfortable for the patient, delivers a higher radiation dose than does scintigraphy, and cannot assess physiology. If radiographic sialography will be performed along with scintigraphic imaging, the radiographic procedure should be performed last as its invasiveness may invoke an inflammatory response. ${ }^{67} \mathrm{Ga}$ scanning has occasionally been used to assess salivary gland function, in the setting of Sjogren syndrome for example. CT and MRI may be most helpful in assessing unknown causes of facial swelling and in locating the anatomic position of masses preoperatively. A higher radiation dose is delivered with CT than with either radiographic or salivary gland scintigraphy; however, CT provides significant detail anatomically. Needle biopsy with the aid of radiography or ultrasound may also be used to determine cell type within a mass.

\section{CONCLUSION}

This article has provided an overview of the clinical and technical aspects of 6 infrequently performed studies in nuclear medicine. This article may be of use to technologists faced with the task of performing one of these uncommon studies.

\section{ACKNOWLEDGMENT}

We thank Dr. Isabelle Savoie for her contribution of the chylothorax case.

\section{REFERENCES}

1. MacDonald A, Burrell S. Infrequently performed studies in nuclear medicine: part 1. J Nucl Med Technol. 2008;36:132-143.

2. Wilson MA. Clinical protocols. In: Wilson MA, ed. Textbook of Nuclear Medicine. Philadelphia, PA: Lippincott-Raven; 1998:571-572.

3. Harbert J, Gonçalves Da Rocha AF. Radionuclide cisternography. In: Harbert J, ed. Textbook of Nuclear Medicine. Vol II: Clinical Applications. 2nd ed. Philadelphia, PA: Lea \& Febiger; 1984:111-126.

4. Shemie SD, Doig C, Dickens B, et al. Brain arrest: the neurological determination of death and organ management in Canada. CMAJ. 2006;174: S1-S30. 
5. Wijdicks EF. Determining brain death in adults. Neurology. 1995;45:1003-1011.

6. Donohoe KJ, Frey KA, Gerbaudo VH, Mariani G, Nagel JS, Shulkin B. Society of Nuclear Medicine Procedure Guideline for Brain Death Scintigraphy. Version 1.0, approved February 25, 2003. Available at: http://interactive.snm.org/docs/ pg_ch20_0403.pdf. Accessed January 21, 2009.

7. Huang AH. The hot nose sign. Radiology. 2005;235:216-217.

8. Wieler H, Marohl K, Kaiser KP, Klawki P, Frosslers H. Tc-99m HMPAO cerebral scintigraphy: a reliable, noninvasive method for determination of brain death. Clin Nucl Med. 1993;18:104-109.

9. Bushnell LB, Perlman SB. Central nervous system. In: Wilson MA, ed. Textbook of Nuclear Medicine. Philadelphia, PA: Lippincott-Raven; 1998: 253-254.

10. Larar GN, Nagel JS. Technetium-99m-HMPAO cerebral perfusion scintigraphy: considerations for a timely brain death declaration. J Nucl Med. 1992;33:22092213.

11. HMPAO (Ceretec/Exametazime) [product monograph]. Mississauga, Ontario, Canada; April 2006. GE Healthcare Canada Inc.

12. Haynes BE, Bessen HA, Haynes VE. The diagnosis of testicular torsion. JAMA. 1983;249:2522-2527.

13. Donohue RE, Utley WL. Torsion of spermatic cord. Urology. 1978;11:33-36.

14. Petrack EM, Hafeez W. Testicular torsion versus epididymitis: a diagnostic challenge. Pediatr Emerg Care. 1992;8:347-350.

15. Beckles MA, Spiro SG, Colice GL, Rudd RM. The physiologic evaluation of patients with lung cancer being considered for resectional surgery. Chest. 2003; 123:105S-114S

16. Parker JA, Coleman RE, Hilson AJW, Royal HD, Siegel BA, Sostman HD. Society of Nuclear Medicine Procedure Guideline for Lung Scintigraphy. Version
3.0, approved February 7, 2004. Available at: http://interactive.snm.org/docs/ Lung\%20Scintigraphy_v3.0.pdf. Accessed January 21, 2009.

17. Draximage MAA [product monograph]. Kirkland, Quebec, Canada; March 2005.

18. Win T, Tasker A, Grooves A, et al. Ventilation-perfusion scintigraphy to predict postoperative pulmonary function in lung cancer patients undergoing pneumonectomy. AJR. 2006;187:1260-1265.

19. Szuba A, Shin WS, Strauss HW, Rockson S. The third circulation: radionuclide lymphoscintigraphy in the evaluation of lymphedema. J Nucl Med. 2003;44: 43-57.

20. Sener SF, Winchester DJ, Martz CH, et al. Lymphedema after sentinel node lymphadenectomy for breast carcinoma. Cancer. 2001;92:748-752.

21. Greyson ND. Salivary glands. In: Maisey MN, Britton KE, Collier BD, eds. Clinical Nuclear Medicine. 3rd ed. London, U.K.: Chapman \& Hall; 1998:557566.

22. Schall GL, Larson SM, Anderson LG, Griffith JM. Quantification of parotid gland uptake of pertechnetate using a gamma scintillation camera and a "regionof-interest" system. AJR. 1972;115:689-697.

23. deRossi G, Calcagni ML. Salivary gland disorders. In: Murray IPC, Ell PJ, eds. Nuclear Medicine in Clinical Diagnosis Treatment: Volume 1. New York, NY: Churchill Livingstone; 1994:373-376.

24. Loutfi I, Nair MK, Ebrahim AK. Salivary gland scintigraphy: the use of semiquantitative analysis for uptake and clearance. J Nucl Med Technol. 2003;31:81-85.

25. Miyake H, Matsumoto A, Hori Y, et al. Warthin's tumor of parotid gland on Tc99m pertechnetate scintigraphy with lemon juice stimulation: Tc-99m uptake, size, and pathologic correlation. Eur Radiol. 2001;11:2472-2480. 